\title{
EQUIVALENCE OF NONLINEAR SYSTEMS TO INPUT-OUTPUT PRIME FORMS ${ }^{*}$
}

\author{
R. MARINO ${ }^{\dagger}$, W. RESPONDEK ${ }^{\ddagger}$, AND A. J. VAN DER SCHAFT ${ }^{\S}$
}

\begin{abstract}
The problem of transforming nonlinear control systems into input-output prime forms is dealt with, using state space, static state feedback, and also output space transformations. Necessary and sufficient geometric conditions for the solvability of this problem are obtained. The results obtained generalize well-known results both on feedback linearization as well as input-output decoupling of nonlinear systems. It turns out that, from a computational point of view, the output space transformation is the crucial step, that is performed by constructing rectifying coordinates for a nested sequence of distributions on the output manifold.
\end{abstract}

Key words. equivalence, output transformation, input-output prime system, integrable distributions, inputoutput decoupling

AMS subject classifications. 93C10, 93B17, 58A30

1. Introduction. We consider smooth (i.e., $C^{\infty}$ ) nonlinear systems, depending in an affine way on the inputs $u_{1}, \ldots, u_{m}$, and having $m$ outputs $y_{1}, \ldots, y_{m}$

$$
\dot{x}=f(x)+\sum_{j=1}^{m} g_{j}(x) u_{j}, \quad u=\left(u_{1}, \ldots, u_{m}\right) \in \mathbb{R}^{m},
$$

$$
y_{j}=h_{j}(x), \quad j=1, \ldots, m
$$

where $x=\left(x_{1}, \ldots, x_{n}\right)$ and $y=\left(y_{1}, \ldots, y_{m}\right)$ are local coordinates for the state space manifold $M$ and for the output space manifold $Y$, respectively. We assume throughout the exisxtence of an equilibrium point $x_{0} \in M$ such that $f\left(x_{0}\right)=0$ and $h\left(x_{0}\right)=0$. (All results can be adapted to the case $f\left(x_{0}\right) \neq 0$ and/or $h\left(x_{0}\right) \neq 0$; see Remark 2 after the proof of Theorem 6.) Our analysis will be mainly of a local nature (see, however, Theorem 10 and Corollary 11 for global extensions), i.e., we firstly study the system in neighborhoods $V_{x_{0}} \subset M$ and $W_{y_{0}} \subset Y$, where $y_{0}=h\left(x_{0}\right)$. We also assume throughout that $M$ and $Y$ are connected, and that rank $d h(x)$, with $h=\left(h_{1}, \ldots, h_{m}\right)$, equals $m$ in $V_{x_{0}}$, and that the dimension of the distribution $G(x):=\operatorname{span}\left\{g_{1}(x), \ldots, g_{m}(x)\right\}$ is $m$ in $V_{x_{0}}$. Note that we are restricting ourselves entirely to square systems, i.e., the number of inputs equals the number of outputs.

We address the (local) equivalence of $\Sigma$ to prime (linear) systems, and to input-output prime (linear) systems. We use the following notion of equivalence.

DEFINITION 1. Consider two systems $\Sigma_{1}, \Sigma_{2}$ defined on $\left(M_{1}, Y_{1}\right),\left(M_{2}, Y_{2}\right)$ with equilibrium points $x_{01} \in M_{1}, x_{02} \in M_{2}$, respectively. We say that $\Sigma_{1}$ is locally equivalent to $\Sigma_{2}$, around $x_{01}$ and $x_{02}$, if there exist:

(i) Neighborhoods $V_{x_{01}} \subset M_{1}, V_{x_{02}} \subset M_{2}$ and a diffeomorphism $\varphi: V_{x_{01}} \rightarrow V_{x_{02}}$ satisfying $\varphi\left(x_{01}\right)=x_{02}$;

\footnotetext{
* Received by the editors June 24, 1991; accepted for publication (in revised form) June 15, 1992.

† Dipartimento di Ingegneria Elettronica, Seconda Università di Roma, “Tor Vergata," Via O. Raimondo, 00173 Roma, Italy.

$\ddagger$ Institute of Mathematics, Polish Academy of Sciences, Sniadeckich 8, 00-950 Warsaw, Poland. This author’s work was performed while a visiting professor at the Dipartimento di Ingegneria Elettronica, II Università di Roma "Tor Vergata."

$\S$ Department of Applied Mathematics, University of Twente, P.O. Box 217, 7500 AE Enschede, the Netherlands.
} 
(ii) a nonsingular state feedback $u=\alpha(x)+\beta(x) v$ defined on $V_{x_{01}}$ such that $\alpha\left(x_{01}\right)=$ 0 and det $\beta(x) \neq 0$;

(iii) neighborhoods $W_{y_{01}} \subset Y_{1}, W_{y_{02}} \subset Y_{2}$ of $y_{01}=h^{1}\left(x_{01}\right)$ and $y_{02}=h^{2}\left(x_{02}\right)$, where $h^{1}$ and $h^{2}$ denote the output maps of $\Sigma_{1}$ and $\Sigma_{2}$ respectively, and a diffeomorphism $\psi: W_{y_{01}} \rightarrow W_{y_{02}}$ satisfying $\psi\left(y_{01}\right)=y_{02}$,

such that the transformation of $\Sigma_{1}$ under $(\varphi,(\alpha, \beta), \psi)$ equals $\Sigma_{2}$ on the specified neighborhoods.

We recall from [Mo] (see also [He]) the notion of linear prime system.

DEFINITION 2. A system $\Sigma$ is called a (linear) prime system if it is of the form

$$
\begin{aligned}
y_{i} & =x_{i 1} \\
(P) \dot{x}_{i 1} & =x_{i 2} \quad i=1, \ldots, m \\
& \vdots \\
\dot{x}_{i \kappa_{i}} & =u_{i}
\end{aligned}
$$

where $x=\left(x_{11}, \ldots, x_{1 \kappa_{1}}, \ldots, x_{m 1}, \ldots, x_{m \kappa_{m}}\right) \in M=\mathbb{R}^{n}, n=\sum_{i=1}^{m} \kappa_{i}$, for some integers $\kappa_{1}, \ldots, \kappa_{m}$, and $y=\left(y_{1}, \ldots, y_{m}\right) \in Y=\mathbb{R}^{m}$. The integers $\kappa_{1}, \ldots, \kappa_{m}$ equal the orders of the zeros at infinity of the system or the relative degrees, as well as the controllability or observability indices.

More generally we define input-output prime systems.

DEFINITION 3. A system $\Sigma$ is called an input-output prime system if it is of the form

$$
\begin{aligned}
& y_{i}=x_{i 1} \\
& (I-O-P) \quad \dot{x}_{i 1}=x_{i 2} \quad i=1, \ldots, m \\
& \dot{x}_{i \mu_{i}}=u_{i} \\
& \dot{z}=a(z, x)+\sum_{j=1}^{m} b_{j}(z, x) u_{j}, \quad a\left(z_{0}, 0\right)=0
\end{aligned}
$$

where $y=\left(y_{1}, \ldots, y_{m}\right) \in Y=\mathbb{R}^{m}$, and where the state space manifold $M$ has the following special structure. There exists a surjective submersion $\pi: M \rightarrow \mathbb{R}^{\mu}, \mu:=$ $\sum_{i=1}^{m} \mu_{i}$, with $x=\left(x_{11}, \ldots, x_{m \mu_{m}}\right) \in \mathbb{R}^{\mu}$, and $z$ being complementary local coordinates for $M$. The integers $\mu_{1}, \ldots, \mu_{m}$ equal the orders of the zeros at infinity or the relative degrees of the system, as well as the observability indices.

Remark. Observe that the relative degrees are not invariant in our problem because we allow for output transformations (see the Example preceeding Algorithm 7); nor are the observability indices since they can be changed by feedback. However, the structure at infinity does remain unchanged under the considered transformations, and thus this is the right concept to describe the $\mu_{i}$ 's as invariants in our problem. Here, the structure of infinity can be defined either geometrically using the $V^{*}$-algorithm [NS], [1s2], or by means of dynamic extension $[M]$ since, for input-output prime systems (and their equivalents), both definitions coincide. 
We will also be interested in input-output prime systems of special form

$$
\begin{aligned}
y_{i} & =x_{i 1} \\
(I-O-P-S) \dot{x}_{i 1} & =x_{i 2} \quad i=1, \ldots, m \\
& \vdots \\
\dot{x}_{i \mu_{i}} & =u_{i} \\
\dot{z} & =a(z, y)
\end{aligned}
$$

with the same specifications as in Definition 3, the difference being that the $z$-dynamics are only driven by the outputs $y=\left(y_{1}, \ldots, y_{m}\right)$.

The main results of the paper are concerned with identifying, via necessary and sufficient geometric conditions, those nonlinear systems $\Sigma$ which are locally equivalent to prime systems (Theorem 4), to input-output prime systems (Theorem 6), and to input-output prime systems of special form (Proposition 8). Theorem 10 and Corollary 11 deal with global equivalence issues. The results obtained generalize well-known results both on normal forms for input-output decouplable systems as well as on feedback linearization of systems with no outputs, as we will now briefly indicate.

If outputs are not considered in $\Sigma$, and therefore output change of coordinates (iii) is omitted in Definition 1, the problem of local equivalence with prime systems becomes the well-known local feedback linearization problem, i.e., local feedback equivalence into linear (Brunovsky) canonical forms

$$
\dot{x}_{i 1}=x_{i 2}
$$

$$
\begin{gathered}
\vdots \\
\dot{x}_{i \kappa_{i}}=u_{i}
\end{gathered} \quad i=1, \ldots, m,
$$

which was completely solved in [JR] and [HSM]. The solution to this problem is a generalization of a linear result of Brunovsky $[\mathrm{Br}]$, stating that any controllable linear system

$$
\dot{x}=A x+B u, \quad x \in \mathbb{R}^{n}, u \in \mathbb{R}^{m}, \quad \text { rank } B=m,
$$

can be transformed into $(B)$ by the action of the linear feedback group taking the pair $(A, B)$ into $\left(T(A+B F) T^{-1}, T B G\right)$ for a linear state space change of coordinates $\tilde{x}=T x$ and a linear feedback $u=F x+G v, \operatorname{det} G \neq 0$. The set of indices $\left(\kappa_{1}, \ldots, \kappa_{m}\right)$, called controllability indices, is uniquely associated with (6) and forms a complete set of invariants under the action of the linear feedback group (see also [Wo]). In [Mo] Morse enlarges this group by allowing also for linear output space change of coordinates $\tilde{y}=H y$, and gives necessary and sufficient conditions for a linear system

$$
\dot{x}=A x+B u, \quad x \in \mathbb{R}^{n}, \quad u \in \mathbb{R}^{m}, \quad y \in \mathbb{R}^{m},
$$

$$
y=C x, \quad \operatorname{rank} B=\operatorname{rank} C=m,
$$

to be transformed into a prime system $(P)$ given by (1.2) by the action of the group taking $(A, B, C)$ into $\left(T(A+B F) T^{-1}, T B G, H C T^{-1}\right)$. We generalize this result of Morse to nonlinear systems $\Sigma$ in Theorem 4 , on the basis of the local feedback linearization theorem [JR], [HSM]. We remark that nonlinear output change of coordinates was introduced in $[\mathrm{KR}]$ in the study of asymptotic observers. Furthermore, the problem of local feedback 
equivalence (with no output change of coordinates) of $\Sigma$ to a linear system was studied and solved in [CIRT].

The problem of (local) feedback equivalence, without output change of coordinates, of a nonlinear system $\Sigma$ to an input-output prime system $I-O-P$ has been solved in [IKGM]. Indeed, this problem amounts to the (local) nonlinear input-output decoupling problem, as dealt with in [SR], [Fr], and [Si]. The basic tool is the decoupling matrix, which generalizes to nonlinear systems the Falb-Wolovich matrix [FW], used in inputoutput decoupling of linear systems (L). In fact a necessary and sufficient condition for $\Sigma$ to be input-output decouplable around $x_{0}$ is that its decoupling matrix is nonsingular in a neighborhood of $x_{0}$. We note that the problem of local equivalence of $\Sigma$ to $(I-O-P)$ studied in the present paper can be rephrased in this latter terminology as finding a (local) output transformation $\tilde{y}=\psi(y)$ such that $\Sigma$, with the resulting transformed output functions $\tilde{h}_{1}=\psi_{1} o h, \ldots, \tilde{h}_{m}=\psi_{m} o h$, is locally input-output decouplable. Finally, (local and global) feedback equivalence with no output change of coordinates of $\Sigma$ into input-output prime systems of special form $(I-O-P-S)$ has been dealt with in [BI], while for linear systems $(L)$ equivalence to $(I-O-P)$ implies equivalence to $(I-O-P-S)$, as was implicitly derived in [Mo] (see Remark 2 after Proposition 8).

The results obtained are useful for control applications in the following sense. It is well known (see, e.g., [Is], [NvdS]) that many nonlinear control problems are relatively easily attacked for input-output decouplable systems. Now, in many of these control problems output transformations are naturally allowed, and thus our results enable us to treat in a similar way a class of nonlinear control systems which properly contains the input-output decouplable systems. One obvious example of a control problem which naturally does allow for output transformations is the (asymptotic) output tracking problem by static state feedback control (see the example after Theorem 6).

2. Main results. Let us first recall the definitions of the following sequences of distributions for a nonlinear system $\Sigma$ :

$$
\begin{aligned}
G_{1} & :=G:=\operatorname{span}\left\{g_{1}, \ldots, g_{m}\right\} \\
G_{i+1} & :=G_{i}+\left[f, G_{i}\right], \quad i=1,2, \ldots
\end{aligned}
$$

$$
\begin{aligned}
S_{1} & :=G, \\
S_{i+1} & :=S_{i}+\left[f, S_{i} \cap \operatorname{ker} d h\right]+\sum_{j=1}^{m}\left[g_{j}, S_{i} \cap \operatorname{ker} d h\right], \quad i=1,2, \ldots \\
S^{*} & :=\bigcup_{i \geq 1} S_{i} .
\end{aligned}
$$

The distributions $G_{i}$ were introduced in [JR] in the study of the feedback linearization problem, while the algorithm (2.2) and the definition of $S^{*}$ is taken from [IKGM] (with the difference that, in [IKGM], $S_{i}$ in the right-hand side of (2.2) is replaced by its involutive closure; see, however, conditions (i), (iii) of Theorem 4). $S^{*}$, the smallest conditioned invariant distribution containing $G$, enjoys the property (see [IKGM])

$$
\begin{aligned}
{\left[f, S^{*} \cap \operatorname{ker} d h\right] } & \subset S^{*}, \\
{\left[g_{j}, S^{*} \cap \operatorname{ker} d h\right] } & \subset S^{*}, \quad j=1, \ldots, m,
\end{aligned}
$$

and is a generalization of the notion of the smallest conditioned invariant subspace containing $\operatorname{Im} B$, as introduced in [BM] for a linear system (L). If the distributions $S_{i}, i=0,1, \ldots$, all have constant dimension, then there exists an integer $i^{*} \leq n$ such that $S_{i^{*}}=S^{*}$. 
Following [IKGM] we also recall the construction (if it exists) of $V^{*}$, the largest locally controlled invariant distribution contained in kerdh (see also [Hi], and for the linear case [Wo], [BM]). Define the sequence of codistributions

$$
\begin{aligned}
P_{1} & :=d h \\
P_{i+1} & :=P_{i}+L_{f}\left(P_{i} \cap \operatorname{ann} G\right)+\sum_{j=1}^{m} L_{g_{j}}\left(P_{i} \cap \operatorname{ann} G\right), \quad i=1,2, \ldots \\
P^{*} & :=\bigcup_{i \geq 1} P_{i}
\end{aligned}
$$

where $L_{f}, L_{g_{j}}$ denote Lie derivatives, and ann $G$ is the codistribution annihilating $G$. Then the distribution $V^{*}$ is the kernel of the codistribution $P^{*}$, i.e., $V^{*}=\operatorname{ker} P^{*}$ (see, e.g., [Is], [NvdS]).

We finally recall the definition of characteristic indices (or relative degrees) $\rho_{i}$, and of the decoupling matrix. For $i=1, \ldots, m, \rho_{i}$ is defined by

$$
\begin{aligned}
& L_{g_{j}} L_{f}^{k} h_{i}(x)=0, \quad k=0,1, \ldots, \rho_{i}-2, j=1, \ldots, m, \quad \text { for all } x \in V_{x_{0}} \\
& L_{g_{j}} L_{f}^{\rho_{i}-1} h_{i}(x) \neq 0, \quad \text { for some } j \in\{1, \ldots, m\} \quad \text { and } x \in V_{x_{0}} .
\end{aligned}
$$

If $\rho_{i}<\infty, i=1, \ldots, m$, the decoupling matrix $D(x)$ is defined as

$$
D(x)=\left(L_{g_{j}} L_{f}^{\rho_{i}-1} h_{i}(x)\right)_{i, j=1, \ldots, m} .
$$

We now come to our first main theorem.

THEOREM 4. Consider a nonlinear system $\Sigma$ with equilibrium $x_{0} . \quad \Sigma$ is locally equivalent to a prime system $(P)$ with equilibrium 0 , if and only if the following conditions are satisfied in a neighborhood of $x_{0}$ :

(i) $G_{i}$ is involutive and of constant dimension, $i=1, \ldots, n-1$;

(ii) $G_{n}=T M$;

(iii) $G_{i}=S_{i}, i=1,2, \ldots, n$;

(iv) $G_{i}+\operatorname{ker} d h$ is involutive and of constant dimension, $i=1, \ldots, n-1$.

Remark 1. Theorem 4 generalizes and clarifies the following result of Morse ([Mo, Thm. 3.1]): The system (L), i.e., the triple $(A, B, C)$, is transformable by $(T,(F, G), H)$ : $(A, B, C) \rightarrow\left(T(A+B F) T^{-1}, T B G, H C T^{-1}\right)$ into a prime system $(P)$ if and only if:

(i) $V^{*}=0$

(ii) $G_{n}=\operatorname{Im}\left(B, A B, \ldots, A^{n-1} B\right)=\mathbb{R}^{n}$;

(iii) ${ }^{\prime} G_{i}=S_{i}, i=1, \ldots, n$.

Conditions (i) and (iv) of Theorem 4 are always satisifed for linear systems, while they are crucial integrability conditions in the nonlinear case. Conditions (ii)' and (iii)' of Morse are specializations of conditions (ii) and (iii) of Theorem 4 to the linear case. Condition (i), i.e., $V^{*}=0$, is redundant; it is implied by conditions (ii) and (iii). In fact the proof that we will give is entirely different from Morse's and enables us to point out the redundancy of the condition $V^{*}=0$ in the original statement of Morse.

Remark 2. Conditions (i) and (ii) are the necessary and sufficient conditions given in [JR] for the system $\Sigma$ without outputs to be locally feedback equivalent to a linear system in Brunovsky form (B).

Remark 3. While Remarks 1 and 2 clarify the necessity of conditions (i), (ii), and (iii), we may wonder if condition (iv) is not redundant, since already condition (iii) enforces a rather strong compatibility between $G_{i}$ and kerdh. However, the following example shows 
that for $\rho>2$ condition (iv) is not implied by conditions (i), (ii), and (iii). Consider the system

$$
\begin{array}{ll}
\dot{x}_{1}=u_{1}, & y_{1}=x_{1} \\
\dot{x}_{2}=x_{3}, & y_{2}=x_{2}+x_{1} x_{5} \\
\dot{x}_{3}=u_{2} & \\
\dot{x}_{4}=x_{5}, & y_{3}=x_{4} \\
\dot{x}_{5}=x_{6} & \\
\dot{x}_{6}=u_{3} . &
\end{array}
$$

We easily compute

$$
\begin{gathered}
G_{1}=S_{1}=\operatorname{span}\left\{\frac{\partial}{\partial x_{1}}, \frac{\partial}{\partial x_{3}}, \frac{\partial}{\partial x_{6}}\right\} \\
\text { ker } d h=\operatorname{span}\left\{\frac{\partial}{\partial x_{3}}, \frac{\partial}{\partial x_{5}}-x_{1} \frac{\partial}{\partial x_{2}}, \frac{\partial}{\partial x_{6}}\right\} \\
G_{2}=S_{2}=\operatorname{span}\left\{\frac{\partial}{\partial x_{1}}, \frac{\partial}{\partial x_{2}}, \frac{\partial}{\partial x_{3}}, \frac{\partial}{\partial x_{5}}, \frac{\partial}{\partial x_{6}}\right\} \\
G_{3}=S_{3}=T \mathbb{R}^{6} \\
G_{1}+\operatorname{ker} d h=\operatorname{span}\left\{\frac{\partial}{\partial x_{1}}, \frac{\partial}{\partial x_{3}}, \frac{\partial}{\partial x_{5}}-x_{1} \frac{\partial}{\partial x_{2}}, \frac{\partial}{\partial x_{6}}\right\}
\end{gathered}
$$

and thus conditions (i), (ii), and (iii) are satisfied, while condition (iv) fails since $G_{1}+$ kerdh is not involutive; in fact,

$$
\left[\frac{\partial}{\partial x_{1}}, \frac{\partial}{\partial x_{5}}-x_{1} \frac{\partial}{\partial x_{2}}\right]=-\frac{\partial}{\partial x_{2}} \notin G_{1}+\operatorname{ker} d h .
$$

It follows that (2.7) is not locally equivalent to a prime system $(P)$.

Remark 4. It is easy to see that if a nonlinear system $(\Sigma)$ with equilibrium $x_{0}$ is locally equivalent to a prime system, then its linearization at $x_{0}$, namely, $\left(\bar{x}=x-x_{0}, \bar{y}=y-h\left(x_{0}\right)\right)$

$$
\begin{aligned}
& \dot{\bar{x}}=\frac{\partial f}{\partial x}\left(x_{0}\right) \bar{x}+\sum_{j=1}^{m} g_{i}\left(x_{0}\right) u_{j} \\
& \bar{y}=\frac{\partial h}{\partial x}\left(x_{0}\right) \bar{x}
\end{aligned}
$$

is also equivalent to a prime system. The converse may be false as the following example shows:

$$
\begin{aligned}
& \dot{x}_{1}=u_{1}, \quad y_{1}=x_{1} \\
& \dot{x}_{2}=x_{3}+\left(1-e^{x_{3}}\right) u_{1}, \quad y_{2}=x_{2} \\
& \dot{x}_{3}=u_{2} .
\end{aligned}
$$

In this case the distribution $G_{1}=\operatorname{span}\left\{\left(\partial / \partial x_{1}\right)+\left(1-e^{x_{3}}\right)\left(\partial / \partial x_{2}\right),\left(\partial / \partial x_{3}\right)\right\}$ is not involutive, so that condition (i) of Theorem 4 is violated. On the other hand the system linearized at the origin is obviously a prime system.

Before giving the proof of Theorem 4, we first recall a lemma which clarifies the meaning of condition (iv). Let $h: M \rightarrow Y$ be such that $\operatorname{rank} d h(x)=m=\operatorname{dim} Y$ on a neighborhood $V_{x_{0}}$. Then $W_{y_{0}}:=h\left(V_{x_{0}}\right)$ is a neighborhood of $y_{0}=h\left(x_{0}\right)$ in $Y$. 
Furthermore let $D$ be a distribution on $M$. Then $D$ is said to be projectable by $h$ on $V_{x_{0}}$ if, for all $x_{1}, x_{2}$ in $V_{x_{0}}$, we have

$$
h\left(x_{1}\right)=h\left(x_{2}\right) \Rightarrow \frac{\partial h}{\partial x}\left(x_{1}\right)\left(D\left(x_{1}\right)\right)=\frac{\partial h}{\partial x}\left(x_{2}\right)\left(D\left(x_{2}\right)\right) .
$$

If $D$ is projectable by $h$ on $V_{x_{0}}$, then we define $h_{*} D$ as the following distribution on $W_{y_{0}}$ :

$$
\left(h_{*} D\right)(y)=\partial h / \partial x(x)(D(x)), \quad \text { with } x \in h^{-1}(y) \cap V_{x_{0}}, \quad y \in W_{y_{0}} .
$$

(For the problem of projecting distributions see also [J].)

LEMMA 5. Let $h$ be such that $\operatorname{rank} d h(x)=\operatorname{dim} Y$ on $V_{x_{0}}$, and let $D$ be involutive and constant dimensional on $V_{x_{0}}$. Then $D$ is projectable by $h$ in $V_{x_{0}}$ to a constant dimensional and involutive distribution $h_{*} D$ on $W_{y_{0}}$ if and only if $D+\operatorname{ker} d h$ is involutive and of constant dimension on $V_{x_{0}}$.

Proof. First notice that by the Rank Theorem [Sp] we can take local coordinates $x=\left(x^{1}, x^{2}\right)$ on $V_{x_{0}}$ such that $h\left(x^{1}, x^{2}\right)=x^{1}$. Then it follows from [vdS] (see also [NvdS, Lem. 14.3]) that if $D+\operatorname{ker} d h$ is involutive and of constant dimension then $D$ is projectable and $h_{*} D$ is involutive and of constant dimension. Conversely (see [vdS]) it follows trivially that if $D$ is projectable then $D+\operatorname{ker} d h$ is involutive (indeed $D+\operatorname{ker} d h$ is of the form span $\left\{k\left(x^{1}\right)\left(\partial / \partial x^{1}\right)+\operatorname{span}\left\{\partial / \partial x^{2}\right\}\right.$ for some $\left.k\right)$. Furthermore, if $h_{*} D$ is constant dimensional then $D+\operatorname{ker} d h$ is constant dimensional.

Proof of Theorem 4 (only if). First we note from (2.2) that the definition of $S_{i}$ is invariant under feedback and output transformations. Suppose that $\Sigma$ is locally equivalent to $(P)$. Clearly, $(P)$ satisfies conditions (i)-(iv). It follows that also the definition of $G_{1}$, and inductively of $G_{i}, i>1$, is invariant under feedback, and thus conditions (i)-(iv) are feedback invariant. Thus we can conclude that conditions (i)-(iv) are also satisfied for $\Sigma$.

(If.) By virtue of (i) and (iii) it follows that $S_{i+1}$ is alternatively given as

$$
S_{i+1}=S_{i}+\left[f, S_{i} \cap \operatorname{ker} d h\right], \quad i=1,2, \ldots
$$

since $\left[g_{j}, S_{i} \cap \operatorname{ker} d h\right]=\left[g_{j}, G_{i} \cap \operatorname{ker} d h\right] \subset G_{i}=S_{i}, j=1, \ldots, m$.

By conditions (i), (iii), (iv) and Lemma 5,

$$
H_{i}:=h_{*} G_{i}=h_{*} S_{i}, \quad i=1, \ldots, n
$$

are well-defined involutive and constant-dimensional distributions on a neighborhood $W_{y_{0}} \subset$ $Y$, while by (ii) $H_{n}=T Y$. Obviously $H_{1} \subset H_{2} \subset \ldots \subset H_{n}$. It follows that we can define integers

$$
\kappa_{1}>\kappa_{2}>\ldots>\kappa_{r}>0
$$

such that

$$
0=H_{1}=\ldots=H_{\kappa_{r}-1} \stackrel{\subsetneq}{\neq} H_{\kappa_{r}}=\ldots=H_{\kappa_{1}-1} \stackrel{\subsetneq}{\neq} H_{\kappa_{1}}=T Y
$$

For ease of notation we will assume that

$$
\operatorname{dim} H_{\kappa_{i}}=\operatorname{dim} H_{\kappa_{i}-1}+1, \quad i=1, \ldots, m
$$

implying that $r=m$, and

$$
\kappa_{1}>\kappa_{2}>\ldots>\kappa_{m}>0, \quad \operatorname{dim} H_{\kappa_{i}}=m-i+1, i=1, \ldots, m .
$$


(Later on we will conclude that $\kappa_{1}, \ldots, \kappa_{m}$ are actually the controllability indices of $\Sigma$.)

Invoking the generalized Frobenius theorem for the nested sequence of distributions $H_{\kappa_{m}} \subset H_{\kappa_{m-1}} \subset \ldots \subset H_{\kappa_{1}}$ (see [JR], [NvdS]), we can choose locally about $y_{0}$ in $W_{y_{0}} m$ independent functions

$$
\psi_{1}, \ldots, \psi_{m}
$$

such that

$$
\begin{aligned}
& \left\langle d \psi_{1}, H_{\kappa_{1}-1}\right\rangle=0 \\
& \left\langle d \psi_{2}, H_{\kappa_{2}-1}\right\rangle=0
\end{aligned}
$$

$(2.19 a)$

$$
\left\langle d \psi_{m-1}, H_{\kappa_{m-1}-1}\right\rangle=0
$$

while

(2.19b)

$$
\begin{aligned}
& \left\langle d \psi_{1}, H_{\kappa_{1}}\right\rangle\left(x_{0}\right) \neq 0 \\
& \left\langle d \psi_{2}, H_{\kappa_{2}}\right\rangle\left(x_{0}\right) \neq 0
\end{aligned}
$$

$$
\left\langle d \psi_{m}, H_{\kappa_{m}}\right\rangle\left(x_{0}\right) \neq 0
$$

In the new local coordinates of the output manifold $Y$, given by

$$
\tilde{y}_{i}:=\psi_{i}(y), \quad i=1, \ldots, m
$$

we obviously have

$$
H_{\kappa_{i}}=\operatorname{span}\left\{\frac{\partial}{\partial \tilde{y}_{m}}, \ldots, \frac{\partial}{\partial \tilde{y}_{i}}\right\}, \quad i=1, \ldots, m
$$

If we define new output functions

$$
\tilde{h}_{i}:=\psi_{i} \text { oh, } \quad i=1, \ldots, m
$$


and recall that $H_{i}=h_{*} G_{i}$ (cf. (2.13)), then (2.19a) yields

$$
\begin{aligned}
& \left\langle d \tilde{h}_{1}, G_{\kappa_{1}-1}\right\rangle=0 \\
& \left\langle d \tilde{h}_{2}, G_{\kappa_{2}-1}\right\rangle=0
\end{aligned}
$$

$$
\left\langle d \tilde{h}_{m-1}, G_{\kappa_{m-1}-1}\right\rangle=0
$$

while by $(2.19 b)$,

$$
\begin{aligned}
& \left\langle d \tilde{h}_{1}, G_{\kappa_{1}}\right\rangle\left(x_{0}\right) \neq 0 \\
& \left\langle d \tilde{h}_{2}, G_{\kappa_{2}}\right\rangle\left(x_{0}\right) \neq 0
\end{aligned}
$$

$$
\left\langle d \tilde{h}_{m}, G_{\kappa_{m}}\right\rangle\left(x_{0}\right) \neq 0
$$

Let us now compute the decoupling matrix $\tilde{D}(x)$ (cf. (2.6)) of $\Sigma$ with the newly defined output functions $\tilde{h}_{1}, \ldots, \tilde{h}_{m}$ (cf. $(2.22)$ ). It readily follows from (2.23), and the Leibniz rule, i.e.,

$$
L_{X}\langle d \varphi, Y\rangle=\left\langle d L_{X} \varphi, Y\right\rangle+\left\langle d \varphi, a d_{X} Y\right\rangle
$$

for any two vector fields $X, Y$ and function $\varphi$, that $\tilde{D}(x)$ is given as (see, e.g., [Is], [NvdS])

$$
\tilde{D}(x)=\left(\begin{array}{c}
(-1)^{\left(\kappa_{1}-1\right)}\left\langle d \tilde{h}_{1}, a d_{f}^{\kappa_{1}-1} g_{1}\right\rangle(x) \ldots(-1)^{\left(\kappa_{1}-1\right)}\left\langle d \tilde{h}_{1}, a d_{f}^{\kappa_{1}-1} g_{m}\right\rangle(x) \\
\vdots \\
(-1)^{\left(\kappa_{m}-1\right)}\left\langle d \tilde{h}_{m}, a d_{f}^{\kappa_{m}-1} g_{1}\right\rangle(x) \ldots(-1)^{\left(\kappa_{m}-1\right)}\left\langle d \tilde{h}_{m}, a d_{f}^{\kappa_{m}-1} g_{m}\right\rangle(x)
\end{array}\right) .
$$

We now make the following claim.

CLAIM. $\tilde{D}(x)$ is nonsingular in a neighborhood $U_{x_{0}}$ of $x_{0} \in M$.

Once this claim has been proved the rest of the proof of Theorem 4 follows easily. Indeed by the theory of input-output decoupling (see, e.g., [IKGM], [Is], [NvdS]) the functions 


$$
\begin{aligned}
& \left(\tilde{h}_{1}, \ldots, L_{f}^{\kappa_{1}-1} \tilde{h}_{1}, \ldots, \tilde{h}_{m}, \ldots, L_{f}^{\kappa_{m}-1} \tilde{h}_{m}\right. \\
& \quad=:\left(x_{11}, \ldots, x_{1 \kappa_{1}}, \ldots, x_{m 1}, \ldots, x_{m \kappa_{m}}\right)
\end{aligned}
$$

are independent on $U_{x_{0}}$, and the state feedback

$$
u=-\tilde{D}^{-1}(x)\left(\begin{array}{c}
L_{f}^{\kappa_{1}} \tilde{h}_{1}(x) \\
\vdots \\
L_{f}^{\kappa_{m}} \tilde{h}_{m}(x)
\end{array}\right)+\tilde{D}^{-1}(x) v
$$

brings the system into the form

$$
\begin{aligned}
\tilde{y}_{i} & =x_{i 1} \\
\dot{x}_{i 1} & =x_{i 2} \quad i=1, \ldots, m, \\
& \vdots \\
\dot{x}_{i \kappa_{i}} & =v_{i} \\
\dot{z} & =a(z, x)+b(z, x) v
\end{aligned}
$$

(where $z \in \mathbb{R}^{n-\left(\kappa_{1}+\ldots+\kappa_{m}\right)}$ are additional coordinates).

Furthermore, it is immediately seen that the $S^{*}$-algorithm (cf. (2.2)) applied to (2.27) yields $\operatorname{dim} S^{*}=\kappa_{1}+\ldots+\kappa_{m}$. Then because of feedback invariance of $S_{i}$ and (ii), i.e., $S_{n}=G_{n}=T M$, it follows that $\kappa_{1}+\ldots+\kappa_{m}=n$, and thus the $z$-part in (2.27) is void, implying that $\Sigma$ with the newly defined output functions $\tilde{h}_{1}, \ldots, \tilde{h}_{m}$ is feedback equivalent to a prime system $(P)$, with controllability indices $\kappa_{1}, \ldots, \kappa_{m}$.

Proof of the claim. We use the following induction argument.

Step 1. Consider $\tilde{h}_{1}$. By (2.23) there exists some $i \in\{1, \ldots, m\}$ such that $\left\langle d \tilde{h}_{1}\right.$, $\left.a d_{f}^{\kappa_{1}-1} g_{i}\right\rangle\left(x_{0}\right) \neq 0$. By relabeling $g_{1}, \ldots, g_{m}$, if necessary, we may thus assume that

$$
\left\langle d \tilde{h}_{1}, a d_{f}^{\kappa_{1}-1} g_{1}\right\rangle\left(x_{0}\right) \neq 0 .
$$

Define the functions $\beta_{i}^{1}(x):=\left\langle d \tilde{h}_{1}, a d_{f}^{\kappa_{1}-1} g_{i}\right\rangle(x), i=1, \ldots, m$, and put locally about $x_{0}$

$$
\tilde{g}_{i}:=g_{i}-\frac{\beta_{i}^{1}}{\beta_{1}^{1}} g_{1}, \quad i=2, \ldots, m
$$

(observe that by $(2.28) \beta_{1}^{1} \neq 0$ locally about $x_{0}$ ). Then, because

$$
a d_{f}^{\kappa_{1}-1} \tilde{g}_{i}=a d_{f}^{\kappa_{1}-1} g_{i}-\frac{\beta_{1}^{1}}{\beta_{1}^{1}} a d_{f}^{\kappa_{1}-1} g_{i}\left(\bmod G_{\kappa_{1}-1}\right)
$$

the transformed input vectorfields $\tilde{g}_{2}, \ldots, \tilde{g}_{m}$ satisfy

$$
\left\langle d \tilde{h}_{1}, a d_{f}^{\kappa_{1}-1} \tilde{g}_{i}\right\rangle \equiv 0, \quad \text { around } x_{0}, \quad i=2, \ldots, m .
$$

For ease of notation we will now omit the tildes above $g_{i}$, and thus denote $\tilde{g}_{2}, \ldots \tilde{g}_{m}$ again by $g_{2}, \ldots, g_{m}$.

Step $k+1$. Assume that the functions $\beta_{i}^{j}:=\left\langle d \tilde{h}_{j}, a d_{f}^{\kappa_{j}-1} g_{i}\right\rangle$ satisfy
(A1) $\beta_{j}^{j}\left(x_{0}\right) \neq 0, \quad j=1, \ldots, k$
(A2) $\beta_{i}^{j} \equiv 0, \quad i=j+1, \ldots, m, \quad j=1, \ldots, k$. 
We will show that, after applying feedback, (A1) and (A2) also hold for $j=k+1$. First we note that since $\left\langle d \tilde{h}_{j}, G_{\kappa_{j}-1}\right\rangle=0$ (cf. (2.23a)) repeated use of the Leibniz rule yields

$$
\left\langle d L_{f}^{\ell} \tilde{h}_{j}, a d_{f}^{\kappa_{j}-\ell-1} g_{i}\right\rangle=(-1)^{\ell} \beta_{i}^{j}, \quad \ell=0,1, \ldots, \kappa_{j}-1, \quad i, j=1, \ldots, m .
$$

By using (A1) and (A2) this implies that we have the following "table" for the expressions $\left\langle d L_{f}^{\kappa_{1}-\kappa_{k}} \tilde{h}_{j}, a d_{f}^{\kappa_{k}-1} g_{i}\right\rangle, j=1, \ldots, k, i=1, \ldots, m$ :

$$
\begin{aligned}
& \begin{array}{cccccc}
a d_{f}^{\kappa_{k}-1} g_{1} & a d_{f}^{\kappa_{k}-1} g_{2} & \ldots & a d_{f}^{\kappa_{k}-1} g_{k} & \ldots & a d_{f}^{\kappa_{k}-1} g_{m} \\
* & & 0 & &
\end{array} \\
& \begin{array}{lllllllll}
d L_{f}^{\kappa_{1}-\kappa_{k}} \tilde{h}_{1} & * & & 0 & \\
d L_{f}^{\kappa_{2}-\kappa_{k}} \tilde{h}_{2} & & * & &
\end{array} \\
& d \tilde{\tilde{h}}_{k}
\end{aligned}
$$

where the * elements are all nonzero by (A1). It follows that the map $F=\left(L_{f}^{\kappa_{1}-\kappa_{k}} \tilde{h}_{1}\right.$, $L_{f}^{\kappa_{2}-\kappa_{k}} \tilde{h}_{2}, \ldots, \tilde{h}_{k}$ ) has rank $k$ (the same argument is used in feedback linearization, cf. [HSM], [Is], [NvdS]) and furthermore, since $G_{\kappa_{k}}+\operatorname{ker} d F=T M$, Lemma 5 implies that $G_{\kappa_{k}}$ is projectable by $F$, while

$$
\operatorname{dim} F_{*} G_{\kappa_{k}}=k .
$$

Now consider $\tilde{h}_{k+1}$. Because of (2.23) it follows that there exists some $i \in\{1, \ldots, m\}$ such that

$$
\left\langle d \tilde{h}_{k+1}, a d_{f}^{\kappa_{k+1}-1} g_{i}\right\rangle\left(x_{0}\right) \neq 0 .
$$

We claim that we can take $i \in\{k+1, \ldots, m\}$ having this property. Indeed, otherwise we would have

$$
\left\langle d \tilde{h}_{k+1}, a d_{f}^{\kappa_{k+1}-1} g_{i}\right\rangle\left(x_{0}\right)=0, \quad i=k+1, \ldots, m .
$$

Now take any $X \in G_{\kappa_{k+1}} \cap \operatorname{ker} d h$, then $X$ is of the form $X=\sum_{i=1}^{n} \alpha_{i} a d_{f}^{\kappa_{k+1}-1} g_{i}+Z, Z \in$ $G_{\kappa_{k+1}-1}$, and with the functions $\alpha_{i}$ satisfying

$$
\begin{aligned}
0 & =\left\langle d \tilde{h}_{k+1}, \sum_{i=1}^{n} \alpha_{i} a d_{f}^{\kappa_{k+1}-1} g_{i}\right\rangle\left(x_{0}\right) \\
& =\sum_{i=1}^{k} \alpha_{i}\left(x_{0}\right) c_{i}, \quad \text { with } c_{i}:=\left\langle d \tilde{h}_{k+1}, a d_{f}^{\kappa_{k+1}-1} g_{i}\right\rangle\left(x_{0}\right)
\end{aligned}
$$

where at least one of the $c_{i}$ 's is unequal to zero because of (2.35). Now

$$
G_{\kappa_{k+1}+1}=S_{\kappa_{k+1}+1}=\left[f, G_{\kappa_{k+1}} \cap \operatorname{ker} d h\right]+G_{\kappa_{k+1}}
$$

and inductively,

$$
G_{\kappa_{k}} \subset a d_{f}^{\kappa_{k}-\kappa_{k+1}}\left(G_{\kappa_{k+1}} \cap \operatorname{ker} d h\right)+G_{\kappa_{k}-1} .
$$

Therefore any element of $G_{\kappa_{k}}$ is of the form

$$
\sum_{i=1}^{m} \alpha_{i} a d_{f}^{\kappa_{k}-1} g_{i}+Z, \quad Z \in G_{\kappa_{k}-1},
$$

with $\alpha_{i}\left(x_{0}\right), i=1, \ldots k$, satisfying (2.37). Hence, because of table (2.33) and the nontrivial relation (2.37), the space $\left(F_{*} G_{\kappa_{k}}\right)\left(F\left(x_{0}\right)\right)$ is at most $(k-1)$ dimensional which is in 
contradiction with (2.34). Therefore there does exist some $i \in\{k+1, \ldots, m\}$ such that (2.35) holds. After reordering, if necessary, $g_{k+1}, \ldots, g_{m}$ we may thus assume that

$$
\left\langle d \tilde{h}_{k+1}, a d_{f}^{\kappa_{k+1}-1} g_{k+1}\right\rangle\left(x_{0}\right) \neq 0 .
$$

Now define $\beta_{i}^{k+1}:=\left\langle d \tilde{h}_{k+1}, a d_{f}^{\kappa_{k+1}-1} g_{i}\right\rangle$ and set $\tilde{g}_{i}:=g_{i}-\left(\beta_{i}^{k+1} / \beta_{k+1}^{k+1}\right) g_{k+1}, i=k+$ $2, \ldots, m$. Then as in Step 1, cf. (2.30), we obtain

$$
\left\langle d \tilde{h}_{k+1}, a d_{f}^{\kappa_{k+1}-1} \tilde{g}_{i}\right\rangle \equiv 0, \quad \text { around } x_{0}, \quad i=k+2, \ldots, m .
$$

Omitting again the tildes above $\tilde{g}_{i}$ we have thus proved that (A1), (A2) also hold for $j=k+1$. Hence by induction we have proved that (A1), (A2) hold for every $k=1, \ldots, m$, for the feedback transformed system (the feedback arising from successively applying Step 1 up to Step m). It immediately follows from expression (2.25) that the decoupling matrix $\tilde{D}\left(x_{0}\right)$ for this feedback transformed is a lower triangular matrix with nonzero diagonal elements $\beta_{1}^{\mathrm{l}}\left(x_{0}\right), \ldots, \beta_{m}^{m}\left(x_{0}\right)$, and thus is nonsingular. Since the rank of the decoupling matrix is invariant under feedback [Is], [NvdS] we have proved the claim.

As we have already remarked (see Remark 2 after Theorem 4), Theorem 4 and its proof are closely related to the local feedback linearization problem [JR], [HSM]. However, we would like to stress that from a computational point of view the transformation of $\Sigma$ into a prime system $(P)$ as given by Theorem 4 may be much simpler than the solution to the local feedback linearization problem. In fact for the latter problem we have to find, in some way or another (see [JR], [HSM]), rectifying (Frobenius) coordinates for the whole sequence of distributions $G_{1} \subset G_{2} \subset \ldots \subset G_{n}=T M$, on the (possibly highdimensional) state space manifold $M$. On the other hand, in order to transform $\Sigma$ into $(P)$ we basically have to find rectifying (Forbenius) coordinates for the projected distributions $H_{1} \subset H_{2} \subset \ldots \subset H_{n}=T Y$ on the output space manifold $Y$. In general the dimension of $Y$ is much smaller than that of $\mathrm{M}$, and therefore, assuming that the projections $H_{1}, \ldots, H_{n}$ are easily computed, the latter problem is likely to be simpler. We defer a more elaborate computational implementation of Theorem 4 until after the proof of the next theorem, which deals with the more general problem of local equivalence to input-output prime systems. Recall that given two distributions $D_{1}, D_{2}$ on $M$ we call $D_{1}$ involutive modulo $D_{2}$ if for any two $X, Y \in D_{1}$ we have $[X, Y] \in D_{1}+D_{2}$. Furthermore, observe that if the codistributions $P_{i}, i=1,2, \ldots$ in (2.4) are constant dimensional then $V^{*}$, the largest locally controlled distribution contained in kerdh, exists and is constant dimensional (and is given as $V^{*}=\operatorname{ker} P^{*}$ ).

THEOREM 6. Consider a nonlinear system $\Sigma$ with equilibrium $x_{0} . \Sigma$ is locally equivalent to an input-output prime system $(I-O-P)$ with equilibrium $\left(0, z_{0}\right)$, if and only if the following conditions are satisfied in a neighborhood of $x_{0}$

(i) $P_{i}$ is constant dimensional $i=1,2, \ldots, n$;

(ii) $G_{i}$ is involutive modulo $V^{*}$, and $G_{i}+V^{*}$ is constant dimensional; $i=1, \ldots, n-$ 1

(iii) $G_{n}+V^{*}=T M$;

(iv) $G_{i}=S_{i}$ modulo $V^{*}, i=1,2, \ldots, n$;

(v) $G_{i}+$ kerdh is involutive and of constant dimension, $i=1,2, \ldots, n-1$.

Proof (only if). Suppose that $\Sigma$ is locally equivalent to $(I-O-P)$. Clearly, $(I-O-P)$ satisfies conditions (i)-(v) (notice that $V^{*}=\operatorname{span}\{\partial / \partial z\}$ ). By (2.2) the definition of $S_{i}$ is invariant under feedback. From the fact that $(I-O-P)$ satisfies condition (ii), it follows that also the definition of $G_{i}$ is invariant under feedback modulo $V^{*}$ (i.e., $G_{i}$ for the feedback transformed system is equal modulo $V^{*}$ to $G_{i}$ for the original system). In 
particular, since $V^{*} \subset$ kerdh, it follows that the definition of $G_{i}+\operatorname{ker} d h$ is invariant under feedback. Thus conditions (i)-(v) are feedback invariant and we can conclude that they are satisfied by $\Sigma$.

(If.) By definition of $V^{*}$ (see, e.g., [IKGM], [Hi]) there exists locally around $x_{0}$ a feedback $u=\alpha(x)+\beta(x) v, \operatorname{det} \beta(x) \neq 0$, such that

$$
\left[\tilde{f}, V^{*}\right] \subset V^{*}, \quad\left[\tilde{g}_{j}, V^{*}\right] \subset V^{*}, \quad j=1, \ldots, m,
$$

where $\dot{x}=\tilde{f}(x)+\sum_{j=1}^{m} \tilde{g}_{j}(x) v_{j}$ denotes the feedback transformed system. Thus locally around $x_{0}$ we can factor out by the distribution $V^{*}$ to obtain a manifold $M^{\prime}$ and a factor system (see [IKGM])

$$
\dot{x}^{\prime}=f^{\prime}\left(x^{\prime}\right)+\sum_{j=1}^{m} g_{j}^{\prime}\left(x^{\prime}\right) v_{j}, \quad x^{\prime} \in M^{\prime},
$$

$$
y_{j}=h_{j}^{\prime}\left(x^{\prime}\right), \quad j=1, \ldots, m,
$$

i.e., around $x_{0}$ we have the projection $\pi: M \rightarrow M^{\prime}$, with $V^{*}=\operatorname{ker} \pi_{*}$. (Since $V^{*} \subset$ $\operatorname{ker} d h, h=\left(h_{1}, \ldots, h_{m}\right)$ can be also factored to a map $h^{\prime}=\left(h_{1}^{\prime}, \ldots, h_{m}^{\prime}\right): M^{\prime} \rightarrow Y$ satisfying $h=h^{\prime} o \pi$.)

Define the distributions $G_{i}^{\prime}$ and $S_{i}^{\prime}$ for the factor system $\Sigma^{\prime}$. It is readily checked that $G_{i}^{\prime}$ and $S_{i}^{\prime}$ satisfy conditions (i)-(iv) of Theorem 4 for the factor system $\Sigma^{\prime}$ and around $x_{0}^{\prime}=\pi\left(x_{0}\right)$. Indeed, observe again that under conditions (ii) and (iv) of Theorem 6 the distributions $G_{i}$ and $S_{i}$ are feedback invariant modulo $V^{*}$. Then it immediately follows that $G_{i}^{\prime}$ and $S_{i}^{\prime}$ satisfy conditions (i)-(iii) of Theorem 4 applied to $\Sigma^{\prime}$. Finally, since $V^{*} \subset \operatorname{ker} d h$ it follows that $G_{i}$ are also feedback invariant modulo kerdh. Thus $\pi_{*}\left(G_{i}+\operatorname{ker} d h\right)=G_{i}^{\prime}+\operatorname{ker} d h^{\prime}$, and it follows from Lemma 2 (applied to the involutive and constant-dimensional distribution $G_{i}+\operatorname{ker} d h$ and the mapping $\pi: M \rightarrow M^{\prime}$ ) that $G_{i}^{\prime}+\operatorname{ker} d h^{\prime}$ is involutive and constant dimensional.

Hence by Theorem $4, \Sigma^{\prime}$ is locally equivalent to a prime system $(P)$ of the form (1.3a) (with $x_{0}^{\prime}=0$, and $\mu_{i}, i=1, \ldots, m$, the controllability indices of $\Sigma^{\prime}$ ). Since the remaining dynamics of $\Sigma$ are of the general form (1.3b) we conclude that $\Sigma$ is locally equivalent to $(I-O-P)$ with equilibrium $x_{0}=\left(0, z_{0}\right)$.

Remark 1. Note that the indices $\mu_{1}, \ldots, \mu_{m}$ are intrinsically defined. Indeed if $\Sigma$ satisfies the conditions of Theorem 6 then $\mu_{1}, \ldots, \mu_{m}$ are the (intrinsically defined) controllability indices of the factor system $\Sigma^{\prime}$, living on $M / V^{*}$. In particular it follows that an input-output prime system $(I-O-P)$ cannot be equivalent to an input-output prime system with different indices $\mu_{1}, \ldots, \mu_{m}$.

Remark 2. If $\Sigma$ satisfies the conditions of Theorem 6 on a neighborhood of a point $\bar{x}$ which is not an equilibrium, then $\Sigma$ will be locally equivalent to an input-output prime system (1.4) with the addition of a constant drift term $f(\bar{x})$. Furthermore, if $f(\bar{x}) \in G(\bar{x})$ then this drift term can be removed by additional feedback. Similarly, if $h(\bar{x}) \neq 0$, then we have to add to the output equation of (1.4a) the constant term $h(\bar{x})$. Of course, this remark already applies to Theorem 4 .

Remark 3. It follows from the proof of Theorem 6 that $h_{*} G_{i}$ is a well-defined distribution on a neighborhood of $y_{0}=h\left(x_{0}\right)$ (i.e., $G_{i}$ is projectable by $h$ on some neighborhood $\left.V_{x_{0}}\right), i=1, \ldots, m$. In fact $h_{*} G_{i}=h_{*}^{\prime} G_{i}^{\prime}$ (with ' denoting the factor system $\Sigma^{\prime}$ ), and the projectability of $G_{i}^{\prime}$ by $h^{\prime}$ to an involutive constant-dimensional distribution on a neighborhood of $y_{0}$ follows by an application of Lemma 5 to $G_{i}^{\prime}$ and $h^{\prime}$. Note, however, that 
Lemma 5 as it stands cannot be directly applied to $G_{i}$ and $h$ (satisfying condition $(v)$ ), since we do not require $G_{i}$ to be involutive and constant dimensional (but only modulo $\left.V^{*}\right)$.

Note that Theorem 6 generalizes the well-known fact that a nonlinear system $\Sigma$ whose decoupling matrix $D(x)$ (cf. (2.6)) has rank $m$ around $x_{0}$ can be transformed by local state space and feedback transformations into (1.3), see, e.g., [IKGM]. Hence Theorem 6 can also be interpreted as giving the necessary and sufficient conditions for finding a local output transformation $\tilde{y}=\psi(y)=\left(\psi_{1}(y), \ldots, \psi_{m}(y)\right)$ such that the decoupling matrix $\tilde{D}(x)$ for the transformed output functions $h_{1}=\psi_{1} o h, \ldots, \tilde{h}_{m}=\psi_{m} o h$ has rank $m$ around $x_{0}$

Example. Consider the following system on $M=\mathbb{R}^{3}, Y=\mathbb{R}^{2}$ :

$$
\begin{array}{ll}
\dot{x}_{1}=u_{2}, & y_{1}=x_{1} \\
\dot{x}_{2}=x_{3}, & y_{2}=x_{2}+\frac{1}{3} x_{1}^{3} \\
\dot{x}_{3}=u_{1} . &
\end{array}
$$

The relative degrees are both 1 , while the decoupling matrix $D(x)$ equals

$$
D(x)=\left(\begin{array}{cc}
0 & 1 \\
0 & x_{1}^{2}
\end{array}\right)
$$

and thus is singular, implying that the system is not input-output decouplable by static state feedback. However it is readily seen that the system satisfies the conditions of Theorem 6 and even of Theorem 4, and in fact we only need the output transformation

$$
\psi_{1}(y)=y_{2}-\frac{1}{3} y_{1}^{3}, \quad \psi_{2}(y)=y_{1}
$$

to bring the system into prime form (1.2), with $\kappa_{1}=1, \kappa_{2}=2$ (being the relative degrees of the transformed system)! Now suppose we want to asymptotically track a desired smooth trajectory $y^{d}(t)=\left(y_{1}^{d}(t), y_{2}^{d}(t)\right), t \geq 0$ for (2.44). Using the above output transformation, such a trajectory is transformed into the new coordinates as $\tilde{y}^{d}(t)=$ $\left(y_{2}^{d}(t)-\frac{1}{3}\left(y_{1}^{d}(t)\right)^{3}, y_{1}^{d}(t)\right), t \geq 0$, and since (2.44) has been transformed into a prime system the tracking problem is simply solved by a control strategy which is linear in the transformed coordinates, namely,

$$
\begin{aligned}
u_{1}= & -K_{11}\left(x_{2}-\left(y_{2}^{d}(t)-\frac{1}{3}\left(y_{1}^{d}(t)\right)^{3}\right)\right. \\
& -K_{12}\left(x_{3}-\left(\dot{y}_{2}^{d}(t)-\left(y_{1}^{d}(t)\right)^{2} \dot{y}_{1}^{d}(t)\right)\right) \\
& +\ddot{y}_{2}^{d}(t)-2 y_{1}^{d}(t)\left(\dot{y}_{1}^{d}(t)\right)^{2}-\left(y_{1}^{d}(t)\right)^{2} \ddot{y}_{1}^{d}(t) \\
u_{2}= & -K_{2}\left(x_{1}-y_{1}^{d}(t)\right)+\dot{y}_{1}^{d}(t)
\end{aligned}
$$

where $K_{2}<0$, and $K_{11}, K_{12}$ are designed in such a way that the polynomial $s^{2}+K_{12} s$ $+K_{11}$ is Hurwitz.

Notice, furthermore, that the conditions of Theorem 6 imply (see, e.g., [NvdS], [Is]) that $\Sigma$ is input-output decouplable by dynamic state feedback. (In the foregoing example, system (2.44) can be dynamically input-output decoupled for the original output functions by pre-integrating the input $u_{2}$ one time). Regarded from this viewpoint, Theorem 6 avoids the addition of extra pre-integrators to the system by allowing instead for output transformations.

The proofs of Theorems 4 and 6 immediately yield the following algorithm to transform $\Sigma$ into a prime or input-output prime system. 
AlgORITHM 7. Consider a nonlinear system $\Sigma$ with equilibrium $x_{0}$, and satisfying, in a neighborhood $V_{x_{0}}$ of $x_{0}$, conditions (i)-(v) of Theorem 6 . Then $\Sigma$ can be transformed into $(I-O-P)$ in the following way.

(a) Compute the distributions $H_{i}:=h_{*} G_{i}$ on $W_{y_{0}}=h\left(V_{x_{0}}\right), i=1,2, \ldots, n-1$. (By Remark 3 above, $H_{i}$ are all well defined, involutive, and constant-dimensional distributions.)

(b) Construct rectifying (Frobenius) coordinates $\psi_{1}, \ldots, \psi_{m}$ (cf. (2.18), (2.19)) defined on a possibly smaller neighborhood of $y_{0}$, for the whole sequence $H_{1} \subset H_{2} \subset \ldots \subset H_{n-1}$. This defines the output space transformation $\psi$ of Definition 1.

(c) Consider the output functions $\tilde{h}_{i}:=\psi_{i}$ oh, $i=1, \ldots, m$, for $\Sigma$. Compute the relative degrees $\mu_{1}, \ldots, \mu_{m}$ for these output functions and the decoupling matrix $\tilde{D}(x)=$ $\left(L_{g_{j}} L_{f}^{\mu_{i}-1} \tilde{h}_{i}(x)\right)_{i, j=1, \ldots, m}$. Necessarily $\mu_{i}<\infty, i=1, \ldots, m$, and rank $D(x)=m$ around $x_{0}$. Define the functions

$$
x_{i j}:=L_{f}^{(j-1)} \tilde{h}_{i}, \quad j=1, \ldots, \mu_{i}, \quad i=1, \ldots, m .
$$

Necessarily these functions are independent around $x_{0}$, while

$$
V^{*}=\operatorname{ker} \operatorname{span}\left\{d x_{i j}, j=1, \ldots, \mu_{i}, \quad i=1, \ldots, m\right\} \text {. }
$$

Choose complementary coordinates $z=\left(z_{1}, \ldots, z_{n},\right)$ around $x_{0}\left(n^{\prime}:=n-\left(\mu_{1}+\ldots+\mu_{m}\right)\right)$. This defines the state space transformation $\varphi$ of Definition 1.

(d) Compute the regular feedback $u=\alpha(x)+\beta(x) v$ around $x_{0}$ as

$$
\alpha(x)=-\tilde{D}^{-1}(x)\left(\begin{array}{c}
L_{f}^{\mu_{1}} \tilde{h}_{1}(x) \\
\vdots \\
L_{f}^{\mu_{m}} \tilde{h}_{m}(x)
\end{array}\right), \quad \beta(x)=\tilde{D}^{-1}(x) .
$$

This defines the feedback transformation required in Definition 1.

Remark 1. Note that $\mu_{1}+\ldots+\mu_{m}=n$ if and only if $V^{*}=0$, in which case $\Sigma$ is locally equivalent to a prime system.

Remark 2. At some occasions it may be more efficient not to check conditions (i)-(v) of Theorem 6 in order to see if $\Sigma$ is locally equivalent to $(I-O-P)$, but instead to apply directly Algorithm 7. If the Algorithm breaks down (e.g., if some distributions $H_{i}$ are not well defined or not involutive, or if $\tilde{D}(x)$ does not have full rank) then $\Sigma$ is not locally equivalent to $(I-O-P)$ (while $\Sigma$ is locally equivalent to $I-O-P$ if Algorithm 7 does work).

Example. As an illustration of the above remark we apply Algorithm 7 to the example

$$
\begin{aligned}
& \dot{x}_{1}=u_{1}, \quad y_{1}=x_{5}+x_{5} x_{3}-\frac{1}{2} x_{5} x_{2}^{2} \\
& \dot{x}_{2}=u_{2}, \quad y_{2}=x_{3}-\frac{1}{2} x_{2}^{2} \\
& \dot{x}_{3}=x_{1}+x_{2} u_{2} \\
& \dot{x}_{4}=x_{2}+x_{1} u_{1} \\
& \dot{x}_{5}=x_{4}-\frac{1}{2} x_{1}^{2} .
\end{aligned}
$$

Following the proof of Lemma 5 we first express the system in local coordinates

$$
\xi_{1}=x_{1}, \quad \xi_{2}=x_{2}, \quad \xi_{3}=x_{3}-\frac{1}{2} x_{2}^{2}, \quad \xi_{4}=x_{4}, \quad \xi_{5}=x_{5}\left(1+x_{3}-\frac{1}{2} x_{2}^{2}\right)
$$


as

$$
\begin{aligned}
& \dot{\xi}_{1}=u_{1}, \quad y_{1}=\xi_{5} \\
& \dot{\xi}_{2}=u_{2}, \quad y_{2}=\xi_{3} \\
& \dot{\xi}_{3}=\xi_{1} \\
& \dot{\xi}_{4}=\xi_{2}+\xi_{1} u_{1} \\
& \dot{\xi}_{5}=\frac{\xi_{5}}{1+\xi_{3}}\left(1+\xi_{1}\right)+\left(\xi_{4}-\frac{1}{2} \xi_{1}^{2}\right)\left(1+\xi_{3}\right) .
\end{aligned}
$$

(a) Simple computations give

$$
\begin{aligned}
& G_{1}=\operatorname{span}\left\{\frac{\partial}{\partial \xi_{1}},+\xi_{1} \frac{\partial}{\partial \xi_{4}}, \frac{\partial}{\partial \xi_{2}}\right\} \\
& G_{2}=\operatorname{span}\left\{\frac{\partial}{\partial \xi_{1}}, \frac{\partial}{\partial \xi_{2}}, \frac{\partial}{\partial \xi_{4}}, \frac{\partial}{\partial \xi_{3}}+\frac{\xi_{5}}{1+\xi_{3}} \frac{\partial}{\partial \xi_{5}}\right\} \\
& G_{3}=T M \\
& H_{1}=0 \\
& H_{2}=\operatorname{span}\left\{\frac{y_{1}}{1+y_{2}} \frac{\partial}{\partial y_{1}}+\frac{\partial}{\partial y_{2}}\right\} \\
& H_{3}=T Y .
\end{aligned}
$$

According to (2.15) the indices are $\kappa_{2}=2, \kappa_{1}=3$.

(b) From (2.18) the rectifying Frobenius coordinates are

$$
\psi_{1}(y)=\frac{y_{1}}{1+y_{2}} \quad \psi_{2}(y)=y_{2}
$$

(c) The transformed output functions $\tilde{h}_{1}, \tilde{h}_{2}$ are

$$
\tilde{h}_{1}=\frac{x_{5}\left(1+x_{3}-\frac{1}{2} x_{2}^{2}\right)}{1+x_{3}-\frac{1}{2} x_{2}^{2}}=x_{5} \quad \tilde{h}_{2}=x_{3}-\frac{1}{2} x_{2}^{2} .
$$

The relative degrees are $\mu_{1}=3, \mu_{2}=2$, while the decoupling matrix is $\tilde{D}=I_{2 \times 2}$. Hence the functions

$$
\begin{aligned}
& z_{1}=\tilde{h}_{1}=x_{5} \\
& z_{2}=L_{f} \tilde{h}_{1}=x_{4}-\frac{1}{2} x_{1}^{2} \\
& z_{3}=L_{f}^{2} \tilde{h}_{1}=x_{2} \\
& z_{4}=\tilde{h}_{2}=x_{3}-\frac{1}{2} x_{2}^{2} \\
& z_{5}=L_{f} \tilde{h}_{2}=x_{1}
\end{aligned}
$$

give the state space transformation $\varphi$ of Definition 1 .

(d) The regular feedback of definition 1 is

$$
\alpha(x)=0 \quad \beta(x)=I .
$$

In fact, in $z$-coordinates we have

$$
\dot{z}_{1}=z_{2}, \quad \dot{z}_{2}=z_{3}, \quad \dot{z}_{3}=u_{2} \quad \dot{z}_{4}=z_{5} \quad \dot{z}_{5}=u_{1},
$$


which is a linear prime system.

The extension of Theorem 6 to local equivalence into input-output prime systems of special form (1.3) reads as follows.

PROPOSITION 8. Consider a nonlinear system $\Sigma$ with equilibrium $x_{0} . \quad \Sigma$ is locally equivalent to an input-output prime system of special form $(I-O-P-S)$, if and only if, on a neighborhood of $x_{0}$, conditions $(i)-(v)$ of Theorem 6 are satisfied and, additionally:

(vi) $V^{*} \cap S^{*}=0$;

(vii) $S^{*}$ is involutive and constant dimensional.

Proof (only if). Suppose $\Sigma$ is locally equivalent to $(I-O-P-S)$. From the (only if) part of Theorem 6 it follows that $\Sigma$ satisfies conditions (i)-(v). Clearly, $(I-O-P-S)$ satisfies conditions (vi) and (vii). Furthermore the definition of $S^{*}$ is feedback invariant, and thus also $\Sigma$ satisfies conditions (vi) and (vii).

(If.) By (iii), (iv), and (vi) we have $V^{*} \oplus S^{*}=T M$. By Theorem $6, \Sigma$ is locally equivalent to $(I-O-P)$, i.e., (1.3). Here $z$ are coordinate functions which are arbitrary except for the fact that they have to be complementary to the coordinate functions $x=$ $\left(x_{11}, \ldots, x_{m \mu_{m}}\right)$; see (2.45). In the present case, however, since $V^{*} \oplus S^{*}=T M$ and $V^{*}$ and $S^{*}$ are involutive and constant dimensional, we can choose $z$ such that span $\mathrm{dz}=$ ann $S^{*}$. Since, by construction, span $\mathrm{dx}=$ ann $V^{*}$, cf. (2.46), we thus have

$$
V^{*}=\operatorname{span}\left\{\frac{\partial}{\partial z}\right\}, \quad S^{*}=\operatorname{span}\left\{\frac{\partial}{\partial x}\right\} .
$$

Then, first of all, since $G=S_{1} \subset S^{*}=\operatorname{span}\{\partial / \partial x\}$, we have in (1.3)

$$
b_{j}(z, x)=0, \quad j=1, \ldots, m .
$$

Second, by definition of $S^{*},\left[f, S^{*} \cap \operatorname{ker} d h\right] \subset S^{*}$, cf. (2.3), and thus, since $f$ is of the form $f=* \partial / \partial x+a(z, x) \partial / \partial z$ and $S^{*}=\operatorname{span}\{\partial / \partial x\}$

$$
\left[* \frac{\partial}{\partial x}+a(z, x) \frac{\partial}{\partial z}, \frac{\partial}{\partial x_{i j}}\right] \subset \operatorname{span}\left\{\frac{\partial}{\partial x}\right\}, \quad j=2, \ldots, \mu_{i}, \quad i=1, \ldots, m .
$$

(Note that kerdh is everything minus span $\left\{\left(\partial / \partial x_{i 1}\right), i=1, \ldots, m\right\}$.) This implies that $a(z, x)$ in (1.3) may only depend on $z$ and $x_{i 1}=y_{i}, i=1, \ldots, m$, and thus (1.4) results.

Remark 1. If conditions (ii) and (iv) in Proposition 8 are replaced by the stronger conditions

(ii) ${ }^{\prime} G_{i}$ is involutive and of constant dimension, $i=1,2, \ldots, n-1$,

(iv) ${ }^{\prime} G_{i}=S_{i}, i=1, \ldots, n$,

then, following [MBE], $a(z, y)$ in (1.4b) will only depend on those (new) output components $y_{i}$ with $i$ such that $\mu_{i}=\max \left\{\mu_{1}, \ldots \mu_{m}\right\}$.

Remark 2. Necessary and sufficient conditions for transforming into (1.4) (without change of output space coordinates) a nonlinear system $\Sigma$ having invertible decoupling matrix have been identified in [BI]: see also [MBE]. Similar conditions were derived, in a different context, in [vdS]. Notice that in the linear case condition (vii) is automatically satisfied. This explains that for a linear system we can always write (even if condition (vi) is not satisfied) the $V^{*}$ dynamics as being only driven by $y$, as follows from the Morse canonical form [Mo].

EXAMPLE. Consider the single input system

$$
\dot{x}_{1}=x_{4}^{3}, \quad y=x_{2}
$$




$$
\begin{aligned}
& \dot{x}_{2}=x_{3}, \\
& \dot{x}_{3}=x_{4} \\
& \dot{x}_{4}=u .
\end{aligned}
$$

Easy computations give:

$$
\begin{aligned}
& V^{*}=\operatorname{span}\left\{\frac{\partial}{\partial x_{1}}\right\}, \quad \operatorname{ker} d h=\operatorname{span}\left\{\frac{\partial}{\partial x_{1}}, \frac{\partial}{\partial x_{3}}, \frac{\partial}{\partial x_{4}}\right\}, \quad S^{*}=\mathbb{R}^{4}, \\
& S^{*} \cap V^{*}=\operatorname{span}\left\{\frac{\partial}{\partial x_{1}}\right\}, \\
& G_{1}=\operatorname{span}\left\{\frac{\partial}{\partial x_{4}}\right\}, \quad G_{2}=\operatorname{span}\left\{\frac{\partial}{\partial x_{4}}, 3 x_{4}^{2} \frac{\partial}{\partial x_{1}}+\frac{\partial}{\partial x_{3}}\right\}, \\
& G_{3}=\operatorname{span}\left\{\frac{\partial}{\partial x_{4}}, 3 x_{4}^{2} \frac{\partial}{\partial x_{1}}+\frac{\partial}{\partial x_{3}}, \frac{\partial}{\partial x_{2}}\right\} .
\end{aligned}
$$

Theorem 4 does not apply since $G_{2}$ is not involutive. Theorem 6 applies, while Proposition 8 does not apply since $V^{*} \cap S^{*} \neq 0$.

Now let us proceed to a global version of the equivalence results we have obtained so far. Instead of requiring that rank $\operatorname{dh}(\mathrm{x})$ and $\operatorname{dim} G(x)$ are equal to $m$ in a neighborhood of $x_{0}$, we will now have to require this on the whole $M$. Then by the Rank Theorem (e.g., [Sp]), $h(M)$ is an open part of $Y$, and without loss of generality, we may restrict to this part of $Y$ and assume that $h: M \rightarrow Y$ is surjective. The global version of Definition 1 reads now as follows.

DEFINITION 9. Consider two systems $\Sigma_{1}, \Sigma_{2}$ defined on $\left(M_{1}, Y_{1}\right),\left(M_{2}, Y_{2}\right)$ with equilibrium points $x_{01} \in M_{1}, x_{02} \in M_{2}$, respectively. $\Sigma_{1}$ is globally equivalent to $\Sigma_{2}$ if there exist:

(i) A diffeomorphism $\varphi: M_{1} \rightarrow M_{2}$, satisfying $\varphi\left(x_{01}\right)=x_{02}$;

(ii) a nonsingular feedback $u=\alpha(x)+\beta(x) v$ on $M_{1}$ with $\alpha\left(x_{01}\right)=0$ and det $\beta(x) \neq 0$

(iii) a diffeomorphism $\psi: Y_{1} \rightarrow Y_{2}$ with $\psi\left(h_{1}\left(x_{01}\right)\right)=h_{2}\left(x_{02}\right)$ such that the resulting transformation of $\Sigma_{1}$ equals $\Sigma_{2}$.

Since Theorem 6 generalizes Theorem 4 we will only give the global version of Theorem 6 , and state as a corollary the global version of Proposition 8.

THEOREM 10. Consider a nonlinear system $\Sigma$ on $(M, Y)$ with equilibrium $x_{0}$, and assume that $h: M \rightarrow Y$ is a surjective submersion and that $\operatorname{dim} G(x)=m$, for all $x \in M$. Suppose that conditions (i)-(v) of Theorem 6 are satisfied on the whole $M$, and that

(A) There exist globally defined independent functions $\psi_{1}, \ldots, \psi_{m}$ on $Y$ which are rectifying coordinates for $H_{1}, \ldots, H_{m}$, i.e., (2.19a) and $(2.19 \mathrm{~b})$ are satisfied for every $x \in M$ (the local existence of $\psi_{1}, \ldots, \psi_{m}$ is already insured by conditions (i)-(v));

then by Algorithm 7(c), (d) $V^{*}$ is globally given by (2.46) and the feedback (2.47) is globally defined. Furthermore, there exists a surjective submersion $\pi: M \rightarrow M^{\prime}$ with ker $\pi_{*}=V^{*}$, while the factor system $\Sigma^{\prime}$, cf. (2.43), is globally defined on $M^{\prime}$.

Assume additionally that

(B) The vectorfields $f^{\prime}$ and $g_{j}^{\prime}, j=1, \ldots, m$, on $M^{\prime}, c f$. (2.43), are complete; then $M^{\prime}$ equals $\mathbb{R}^{\mu}, \mu=\sum_{i=1}^{m} \mu_{i}$, and thus $\Sigma$ is globally equivalent to an input-output system $(I-O-P)$ with equilibrium $\left(0, z_{0}\right)$. Conversely, if $\Sigma$ is globally equivalent to 
$(I-O-P)$ then conditions (i)-(v) of Theorem 6 are satisfied on the whole $M$, and conditions $(A)$ and $(B)$ hold.

Remark. Since the feedback $(\alpha, \beta)$ depends on the choice of $\psi_{1}, \ldots, \psi_{m}$, also condition $(B)$, i.e., the completeness of the modified vectorfields $f^{\prime}, g_{j}^{\prime}, j=1, \ldots, m$, may depend on the choice of $\psi_{1}, \ldots, \psi_{m}$. This is already illustrated by the following very simple example: Consider the system $\dot{x}=u, y=e^{-x}$ on $M=\mathbb{R}$ and $Y=(0, \infty)$. If we take $\psi(y)=\ell n y$ as a global coordinate on $Y$ (which trivially is rectifying, since $H_{1}=T Y$ ) then $\tilde{h}(x)=\psi o h(x)=-x$, and $g^{\prime}=\tilde{g}=-\partial / \partial x$ is complete, implying that the system is globally equivalent to the prime system $\dot{x}=u, y=x$. However if we would take the global rectifying coordinate $\psi(y)=y$, then $g^{\prime}=e^{x} \partial / \partial x$ is not complete, and indeed, since id $:(0, \infty) \rightarrow \mathbb{R}$ is not a diffeomorphism onto $\mathbb{R}$, the system is not globally transformed into a prime system.

Proof. Suppose conditions (i)-(v) are satisfied on $M$, as well as condition $(A)$. Apply Algorithm 7 using the global rectifying coordinates $\psi_{1}, \ldots, \psi_{m}$ on $Y$. Since $V^{*}$ is constant dimensional on the whole $M$ it follows by a slight adaptation of [HK, Thm. 3.9], see also [IKGM], that $V^{*}$ can be globally factored out, i.e., there exists a surjective submersion $\pi: M \rightarrow M^{\prime}$ with $\operatorname{ker} \pi_{*}=V^{*}$, and the feedback transformed dynamics $\tilde{f}, \tilde{g}_{j}, j=1, \ldots, m$ (with $(\alpha, \beta)$ defined by (2.47)) project to dynamics $f^{\prime}, g_{j}^{\prime}, j=1, \ldots, m$ on $M^{\prime}$ (note that, in contrast to [HK, Thm. 3.9], we do not require $\Sigma$ to be accessible; however, condition (iii) of Theorem 6 insures that $\Sigma$ is "accessible modulo $V^{*}$ "). Now assume that condition (B) is satisfied. By the local equivalence of $\Sigma$ with (1.3) it follows that the vectorfields $g_{j}^{\prime}, a d_{f} g_{j}^{\prime}, \ldots, a d_{f}^{\mu_{j}-1} g_{j}^{\prime}, j=1, \ldots, m$, are commuting and complete vectorfields on $M^{\prime}$ (see [Re], [DBE]). It follows that $M^{\prime}=\mathbb{R}^{\mu-k} \times S^{k}$ for some $k \geq 0$. However, since the functions $L_{f}^{j} \tilde{h}_{i}, j=0,1, \ldots, \mu_{i}-1, i=1, \ldots, m$, are global coordinate functions on $M^{\prime}$ necessarily $k=0$ (since $S^{k}$ is compact). Since $y_{j}=x_{j 1}, j=1, \ldots, m$, we also have $Y \simeq \mathbb{R}^{m}$. It follows [Re], [DBE] that $\Sigma^{\prime}$ is globally equivalent to a linear system, and thus that $\Sigma$ is globally equivalent to $I-O-P$. Conversely, if $\Sigma$ is globally equivalent to $(I-O-P)$ then by the (only if) part of Theorem 6 conditions (i)-(v) are satisfied everywhere. Furthermore, clearly $(I-O-P)$ satisfies Conditions $A$ and $B$.

COROLLARY 11. $\Sigma$ is globally equivalent to $(I-O-P-S)$ if and only if, in addition to conditions (i) $-(v)$ and conditions $A$ and $B$ of Theorem 10, conditions (vi), (vii) of Proposition 8 are satisfied on the whole $M$.

Remark. Analogous reasoning on the global equivalence modulo $V^{*}$ to a linear system was used in [MRS]. Similar conditions for the global equivalence of a nonlinear system with invertible decoupling matrix into (1.3) or (1.4) were derived in [BI].

3. Conclusions and final remarks. Necessary and sufficient geometric conditions have been given for transforming nonlinear systems into (input-output) prime form (of special form), locally as well as globally. The main novelty (e.g., as compared to normal forms for input-output decouplable systems) is that we allow for output transformations. Actually, as made explicit in Algorithm 7 (see also the example following it), the output transformation is the crucial step in the whole transformation procedure and involves the simultaneous integration of a nested sequence of distributions on the output space manifold (similar to the integration of distributions on the state space manifold as in the feedback linearization problem). The results obtained are applicable to control problems where output transformations are naturally allowed, such as output tracking, output regulation, (almost) disturbance decoupling [I], [NvdS], [MRS] and the servomechanism problem. The results enable us to treat the class of nonlinear systems equivalent to input-output prime form very much like the well-studied class of input-output decouplable systems. Finally, as we 
have remarked, the use of output transformations may be an alternative to the use of extra pre-integrators for dynamic intput-output decoupling. This raises the problem of how output transformations may be used to minimize the amount of pre-integrators for dynamic input-output decoupling.

Acknowledgment. Witold Respondek is grateful for the warm hospitality and financial support provided by the Dipartimento di Ingegneria Elettronica, Università di Roma, "Tor Vergata."

\section{REFERENCES}

[Br] P. Brunovsky, A classification of linear controllable systems, Kybernetika 6 (1970), pp. 173-188.

[BI] C. I. Byrnes AND A. IsIDORI, Asymptotic stabilization of minimum phase nonlinear systems, IEEE Trans. Automat. Control, AC-36 (1991), pp. 1122-1137.

[BM] G. Basile AND G. Marro, Controlled and conditioned invariant subspaces in linear system theory, J. Optim. Theory Appl. 3 (1969), pp. 306-315.

[CIRT] D. Cheng, A. Isidori, W. Respondek, AND T. J. TARn, Exact linearization of nonlinear systems with outputs, Math. Systems Theory, 21 (1988), pp. 63-83.

[DBE] W. Dayawansa, W. M. Boothby, AND D. L. ElliotT, Global state and feedback equivalence of nonlinear systems, Systems Control Lett., 6 (1984), pp. 229-234.

[Fr] E. FREUND, The structure of decoupled nonlinear systems, Internat. J. Control, 21 (1975), pp. 443-450.

[FW] P. L. FALB AND W. A. Wolovich, Decoupling in the design and synthesis of multivariable control systems, IEEE Trans. Automat. Control, AC-12 (1967), pp. 651-659.

[He] M. Heymann, The prime structure of linear dynamical systems, SIAM J. Control Optim. 10 (1972), pp. 460-469.

[Hi] R. M. HIRSCHORn, (A, B)-invariant distributions and disturbance decoupling of nonlinear systems, SIAM J. Control Optim. 19(1981), pp. 1-19.

[HK] R. Hermann and A. J. Krener, Nonlinear controllability and observability, IEEE Trans. Automat. Control, AC-22 (1977), pp. 728-740.

[HSM] L. R. Hunt, R. Su and G. Meyer, Design for multi-input nonlinear systems, in Diffèrential Geometric Control Theory, R. W. Brockett, R. S. Millman, H. J. Sussmann, eds., Birkhäuser, Boston, 1983, pp. 268-298.

[Is] A. IsidoRI, Nonlinear Control Systems, 2nd ed., Springer-Verlag, New York, 1989.

[Is2] - Nonlinear feedback, structure at infinity and the input-output linearization problem, in Proc. of MTNS'83, Beer Sheva, P. A. Fuhrmann, ed. Lecture Notes in Control and Information Systems 48, Springer-Verlag, Berlin, New York, 1984, pp. 473-493.

[IKGM] A. Isidori, A. J. Krener, C. Gori-Giorgi and S. Monaco, Nonlinear decoupling via feedback: A differential geometric approach, IEEE Trans. Automat. Control, AC-26 (1981), pp. 331-345.

[J] B. JakubCzYK, Feedback linearization of discrete time systems, Systems Control Lett., 9(1987), pp. $411-416$.

[JR] B. Jakubczyk and W. Respondek, On linearization of nonlinear control systems, Bull. Acad. Polon. Sci. Ser. Sci. Math., 28 (1980), pp. 517-522.

[KR] A. J. KRENER AND W. ReSPONDEK, Nonlinear observers with linearizable error dynamics, SIAM J. Control Optim., 23 (1985), pp. 197-216.

[M] C. H. Moog, Nonlinear decoupling and structure at infinity, Math. Control, Signals Systems, 1 (1988), pp. 257-268.

[Mo] A. S. Morse, Structural invariants of linear multivariable systems, SIAM J. Control Optim. 11 (1973), pp. 446-465.

[MBE] R. Marino, W. M. Boothby and D. L. Elliott, Geometric properties of linearizable control systems, Math. Syst. Theory, 18 (1985), pp. 97-123.

[MRS] R. Marino, W. Respondek and A. J. van der Schaft, Almost disturbance decoupling for single-input single-output nonlinear systems, IEEE Trans. Automat. Control, 34 (1989), pp. 1013-1017.

[NS] H. NiJMEIJER AND J. M. SChUMACHER, Zeros at infinity for affine nonlinear control systems, IEEE Trans. Automat. Control, 30 (1985), pp. 566-573.

[NvdS] H. Nijmeijer and A. J. van der Schaft, Nonlinear Dynamical Control Systems, Springer-Verlag, New York, 1990.

[Re] W. RESPONDEK, Global aspects of linearization, equivalence to polynomial forms and decomposition of nonlinear systems, in Algebraic and Geometric Methods in Nonlinear Control Theory, M. Fliess, M. Hazewinkel, eds., D. Reidel, Dordrecht, 1986, pp. 257-284. 
[Si] P. K. Sinha, State feedback decoupling of nonlinear systems, IEEE Trans. Automat. Control, AC-22 (1977), pp. 487-489.

[Sp] M. A. SPIVAK, A Comprehensive Introduction to Differential Geometry, Vol. I, Publish or Perish, Boston, 1970.

[SR] S. N. Singh AND W. J. Rugh, Decoupling in a class of nonlinear systems by state variable feedback, J. Dynamic Systems Meas. Control, 94 (1972), pp. 323-329.

[vdS] A. J. VAN DER SCHAFT, Observability and controllability for smooth nonlinear systems, SIAM J. Control Optim., 20 (1982), pp. 338-354.

[Wo] W. M. Wonнam, Linear Multivariable Control: A Geometric Approach, 3rd ed. Springer-Verlag, New York, 1985. 